\title{
Une période de renouveau
}

\author{
par Bruce Millin
}

$\mathrm{L}$ 'automne sur la côte ouest est ma saison préférée. Les longues et chaudes journées d'été font place à celles plus fraîches et vivifiantes de l'automne. La saison du hockey arrive à grands pas et elle est chargée d'espoir et de possibilités. De nouveaux joueurs, de nouveaux entraîneurs et de nouvelles stratégies entrent en jeu afin de donner à chaque équipe les meilleures chances de victoire. La situation est la même pour la Société canadienne des pharmaciens d'hôpitaux (SCPH). Un nouveau conseil a été constitué au cours de l'assemblée générale annuelle à St. John's, Terre-Neuve-et-Labrador, en août. Des visages connus ont fait place à d'autres et les nouveaux membres du conseil ont bien hâte de travailler au succès de la Société. Je tiens à remercier tous les membres qui quittent le conseil pour leur engagement et leur dévouement envers l'équipe de la SCPH et, tout particulièrement, Doug Sellinger qui a maintenant terminé les trois années de son mandat à la direction.

En matière de stratégies, la SCPH a, au cours de la dernière année, accordé beaucoup de temps et de ressources à la mise à jour de ses lignes directrices sur la préparation des médicaments. L'incident de sous-dosage de produits chimiothérapeutiques qui a eu lieu l'an passé en Ontario et au Nouveau-Brunswick a mis en lumière la nature complexe de l'acte de préparation de produits pharmaceutiques et la nécessité de fournir des balises pour l'ensemble du personnel qui prépare, manipule et entrepose les produits préparés dans les établissements de soins de santé. Un groupe de membres dévoués de la SCPH, sous la direction de Cathy Lyder (coordonnatrice des affaires professionnelles et du service aux membres), a rédigé de nouvelles lignes directrices portant sur la préparation aseptique et non aseptique des médicaments, y compris les produits radiopharmaceutiques et les médicaments dangereux. Le document exhaustif de 232 pages aborde tous les aspects de la préparation des médicaments et il comprend des sections sur la gestion de la qualité, l'entreposage, la distribution, l'externalisation, la tenue de dossiers, la traçabilité et la gestion des rappels.
La sécurité des patients et du personnel hospitalier est le principe directeur de cette révision qui s'appuie sur le travail du Schéma de coopération dans le domaine de l'inspection pharmaceutique (Pharmaceutical Inspection Co-operation Scheme [PIC/S]) (plus particulièrement sur le PIC/S Guide to Good Practices for the Preparation of Medicinal Products in Healthcare Establishments [2014; www.picscheme.org/publication. php?id=8]) et de l'United States Pharmacopeia Convention (USP). Les meilleures pratiques et la littérature actuelle sont intégrées dans tous les volets des nouvelles lignes directrices de la SCPH. Il faut souligner que ce document n'est pas qu'une simple version canadienne des " General Chapter < 795> " et " General Chapter $<797>$ » de l'USP sur la préparation respectivement de produits pharmaceutiques non stériles et stériles (www.usp.org/ usp-healthcare-professionals/compounding). Il s'appuie plutôt sur les connaissances, les compétences et les efforts des personnes qui travaillent dans ce domaine, non seulement des Canadiens, mais d'experts de partout dans le monde, notamment de l'Australie, de l'Europe, du Royaume-Uni et des États-Unis.

Le travail qu'a nécessité la rédaction de ces lignes directrices était considérable. Les bienfaits pour les membres, les services de pharmacie et les organismes réglementaires seront aussi considérables. Ces lignes directrices mettent en valeur ce que la SCPH fait de mieux : rassembler en une équipe des professionnels émérites qui travaillent à une cause commune et identifier les meilleures pratiques pour améliorer la sécurité des patients et les résultats des soins qui leur sont prodigués dans les établissements de soins de santé où nous travaillons.

Bruce Millin, B. Sc. (Pharm.), ACPR, est président et agent de liaison interne pour la Société canadienne des pharmaciens d'hôpitaux. 